\title{
Angewandte
}

Supporting Information

\section{A Water-Bridged H-Bonding Network Contributes to the Catalysis of the SAM-Dependent C-Methyltransferase HcgC}

Liping $\mathrm{Bai}^{+}$, Tristan Wagner ${ }^{+}$, Tao Xu, Xile Hu, Ulrich Ermler, and Seigo Shima*

anie_201705605_sm_miscellaneous_information.pdf 


\section{Supporting Information}

Materials and methods

Figure S1. Active site of the four monomers in the $\mathrm{HcgC}$ homotetramer. .11

Figure S2. The linear compound found in one of the active site of the tetrameric HcgC....12

Figure S3. Active site structure of $\mathrm{HcgC}$ from M. maripaludis in complex with $\mathrm{SAH}$ and pyridinol 2

Figure S4. Comparison of primary structure of $\mathrm{HcgC}$ 14

Figure S5. The active-site structure of HcgC crystallized in the presence of SAM and 2....16

Figure S6. Kinetic data of the wild-type and mutated enzyme .17

Table 1. Statistics of the crystal structure analysis...................................... 18

References for Supplementary information. .19 


\section{Materials and methods}

\section{Materials}

$S$-(5'-adenosyl)-L-methionine (SAM) chloride dihydrochloride and $S$-(5'-adenosyl)-Lhomocysteine (SAH) were purchased from Sigma-Aldrich. Sodium chloride, potassium chloride, potassium hydroxide, magnesium chloride, hydrogen chloride, Tris (hydroxymethyl) aminomethane, methanol, ethanol, kanamycin, 3-( $N$-morpholino)propanesulfonic acid (MOPS), 4-(2-hydroxyethyl)piperazine-1- ethanesulfonic acid (HEPES) were obtained from Roth. Isopropyl $\beta$-D-thiogalactopyranoside (IPTG) was from Thermo. Pyridinol substrate 2 was chemically synthesized. ${ }^{[1]}$

\section{Construction of the expression vector for $\mathrm{HcgC}$ production}

The hcgC gene from Methanococcus maripaludis strain S2 (MMP1498, GenBank accession number NP_988618.1) was used for heterologous production of $\mathrm{HcgC}$ in this work. The $h c g C$ gene was designed and synthesized by Genscript to optimize the codon usage and mRNA structure for expression in Escherichia coli and to have C-terminal extension with His-tag. The synthesized gene was inserted between the NdeI and SalI sites of pET-24b(+). The hcgC sequences are shown below. The start codon is marked with yellow background. The NdeI and SalI recognition sites are indicated with underline and double-underline, respectively. The codon for the mutation in the enzyme variants is indicated with light-blue background.

\section{The wild type HcgC:}

CATATGAACTACGGCATTACCGAAAGCGTGAAAACGACCCGCAGCAAAATCAAA ATCAAAGATATTGTGTCCGATGTGGTGGAAAAGAAAGCGAACGCCATCAAATAT TTTCTGGAAGGCGAAGAATTTAAACAGGCAATTGTGTTTGGCGCTTACCTGTCAG 
GTTCGTATATCGCGTACTCACTGCTGAAAGATTGCGAAGAAGTCATTATCGTGGA CATTCAGCCGCATCTGAAAGATATTCTGTTCAACGACGGTATCAAATTCATGGAT CTGAACAAACTGCAACTGGAACTGCGTAACGGCACCAGCATCAATCCGGATCTG GTGATTGACCTGACGGGTATCGGCGGTGTTAGTCCGGATCTGATTTCCAAATTCA ATCCGAAAGTTCTGATCGTCGAAGATCCGAAAGGCAACCACGACAAAGGTATCT CTAAAATCGATAACACCGACAAACGTCTGTGCGTGGGCGCGAAAAAAGGTGTTC TGAAAACCTATCGCAGCTCTAAATTTAGCAAAACGTCTGGCACCATGACCCTGGT GGTGGATATTATCATGGACTCATGTCGCGAAATTAACGAACTGGATTCGGTTCTG TATACCATCCCGAATCTGAAATACTTTGAGGGTACGGTCTTCCATGAGAAAAACG TGAAAAAATTCCTGACCGAACTGAATATGTCCGCCATTACCGTTAGTTCCATCGA TCACGTCGAATACGAACTGGAAGAAATCCTGTCAAAAAACATCAGCCGTGTGGA CTCGTTCGTGAAAGAATTTETCGAC

\section{The T6V mutant of HcgC:}

CATATGAACTACGGCATTGTGGAAAGCGTGAAAACGACCCGCAGCAAAATCAAA ATCAAAGATATTGTGTCCGATGTGGTGGAAAAGAAAGCGAACGCCATCAAATAT TTTCTGGAAGGCGAAGAATTTAAACAGGCAATTGTGTTTGGCGCTTACCTGTCAG GTTCGTATATCGCGTACTCACTGCTGAAAGATTGCGAAGAAGTCATTATCGTGGA CATTCAGCCGCATCTGAAAGATATTCTGTTCAACGACGGTATCAAATTCATGGAT CTGAACAAACTGCAACTGGAACTGCGTAACGGCACCAGCATCAATCCGGATCTG GTGATTGACCTGACGGGTATCGGCGGTGTTAGTCCGGATCTGATTTCCAAATTCA ATCCGAAAGTTCTGATCGTCGAAGATCCGAAAGGCAACCACGACAAAGGTATCT CTAAAATCGATAACACCGACAAACGTCTGTGCGTGGGCGCGAAAAAAGGTGTTC TGAAAACCTATCGCAGCTCTAAATTTAGCAAAACGTCTGGCACCATGACCCTGGT GGTGGATATTATCATGGACTCATGTCGCGAAATTAACGAACTGGATTCGGTTCTG 
TATACCATCCCGAATCTGAAATACTTTGAGGGTACGGTCTTCCATGAGAAAAACG TGAAAAAATTCCTGACCGAACTGAATATGTCCGCCATTACCGTTAGTTCCATCGA TCACGTCGAATACGAACTGGAAGAAATCCTGTCAAAAAACATCAGCCGTGTGGA CTCGTTCGTGAAAGAATTTGTCGAC

\section{The Y51F mutant of HcgC:}

CATATGAACTACGGCATTACCGAAAGCGTGAAAACGACCCGCAGCAAAATCAAA ATCAAAGATATTGTGTCCGATGTGGTGGAAAAGAAAGCGAACGCCATCAAATAT TTTCTGGAAGGCGAAGAATTTAAACAGGCAATTGTGTTTGGCGCTTTTCTGTCAG GTTCGTATATCGCGTACTCACTGCTGAAAGATTGCGAAGAAGTCATTATCGTGGA CATTCAGCCGCATCTGAAAGATATTCTGTTCAACGACGGTATCAAATTCATGGAT CTGAACAAACTGCAACTGGAACTGCGTAACGGCACCAGCATCAATCCGGATCTG GTGATTGACCTGACGGGTATCGGCGGTGTTAGTCCGGATCTGATTTCCAAATTCA ATCCGAAAGTTCTGATCGTCGAAGATCCGAAAGGCAACCACGACAAAGGTATCT CTAAAATCGATAACACCGACAAACGTCTGTGCGTGGGCGCGAAAAAAGGTGTTC TGAAAACCTATCGCAGCTCTAAATTTAGCAAAACGTCTGGCACCATGACCCTGGT GGTGGATATTATCATGGACTCATGTCGCGAAATTAACGAACTGGATTCGGTTCTG TATACCATCCCGAATCTGAAATACTTTGAGGGTACGGTCTTCCATGAGAAAAACG TGAAAAAATTCCTGACCGAACTGAATATGTCCGCCATTACCGTTAGTTCCATCGA TCACGTCGAATACGAACTGGAAGAAATCCTGTCAAAAAACATCAGCCGTGTGGA CTCGTTCGTGAAAGAATTTGTCGAC

\section{The S175A mutant of HcgC:}

CATATGAACTACGGCATTACCGAAAGCGTGAAAACGACCCGCAGCAAAATCAAA ATCAAAGATATTGTGTCCGATGTGGTGGAAAAGAAAGCGAACGCCATCAAATAT 
TTTCTGGAAGGCGAAGAATTTAAACAGGCAATTGTGTTTGGCGCTTACCTGTCAG GTTCGTATATCGCGTACTCACTGCTGAAAGATTGCGAAGAAGTCATTATCGTGGA CATTCAGCCGCATCTGAAAGATATTCTGTTCAACGACGGTATCAAATTCATGGAT CTGAACAAACTGCAACTGGAACTGCGTAACGGCACCAGCATCAATCCGGATCTG GTGATTGACCTGACGGGTATCGGCGGTGTTAGTCCGGATCTGATTTCCAAATTCA ATCCGAAAGTTCTGATCGTCGAAGATCCGAAAGGCAACCACGACAAAGGTATCT CTAAAATCGATAACACCGACAAACGTCTGTGCGTGGGCGCGAAAAAAGGTGTTC TGAAAACCTATCGCAGCTCTAAATTTAGCAAAACGGCGGGCACCATGACCCTGGT GGTGGATATTATCATGGACTCATGTCGCGAAATTAACGAACTGGATTCGGTTCTG TATACCATCCCGAATCTGAAATACTTTGAGGGTACGGTCTTCCATGAGAAAAACG TGAAAAAATTCCTGACCGAACTGAATATGTCCGCCATTACCGTTAGTTCCATCGA TCACGTCGAATACGAACTGGAAGAAATCCTGTCAAAAAACATCAGCCGTGTGGA CTCGTTCGTGAAAGAATTTETCGAC

\section{The T179V mutant of HcgC:}

CATATGAACTACGGCATTACCGAAAGCGTGAAAACGACCCGCAGCAAAATCAAA ATCAAAGATATTGTGTCCGATGTGGTGGAAAAGAAAGCGAACGCCATCAAATAT TTTCTGGAAGGCGAAGAATTTAAACAGGCAATTGTGTTTGGCGCTTACCTGTCAG GTTCGTATATCGCGTACTCACTGCTGAAAGATTGCGAAGAAGTCATTATCGTGGA CATTCAGCCGCATCTGAAAGATATTCTGTTCAACGACGGTATCAAATTCATGGAT CTGAACAAACTGCAACTGGAACTGCGTAACGGCACCAGCATCAATCCGGATCTG GTGATTGACCTGACGGGTATCGGCGGTGTTAGTCCGGATCTGATTTCCAAATTCA ATCCGAAAGTTCTGATCGTCGAAGATCCGAAAGGCAACCACGACAAAGGTATCT CTAAAATCGATAACACCGACAAACGTCTGTGCGTGGGCGCGAAAAAAGGTGTTC TGAAAACCTATCGCAGCTCTAAATTTAGCAAAACGTCTGGCACCATGGTCCTGGT 
GGTGGATATTATCATGGACTCATGTCGCGAAATTAACGAACTGGATTCGGTTCTG TATACCATCCCGAATCTGAAATACTTTGAGGGTACGGTCTTCCATGAGAAAAACG TGAAAAAATTCCTGACCGAACTGAATATGTCCGCCATTACCGTTAGTTCCATCGA TCACGTCGAATACGAACTGGAAGAAATCCTGTCAAAAAACATCAGCCGTGTGGA CTCGTTCGTGAAAGAATTTGTCGAC

\section{The E209Q mutant of HcgC:}

CATATGAACTACGGCATTACCGAAAGCGTGAAAACGACCCGCAGCAAAATCAAA ATCAAAGATATTGTGTCCGATGTGGTGGAAAAGAAAGCGAACGCCATCAAATAT TTTCTGGAAGGCGAAGAATTTAAACAGGCAATTGTGTTTGGCGCTTACCTGTCAG GTTCGTATATCGCGTACTCACTGCTGAAAGATTGCGAAGAAGTCATTATCGTGGA CATTCAGCCGCATCTGAAAGATATTCTGTTCAACGACGGTATCAAATTCATGGAT CTGAACAAACTGCAACTGGAACTGCGTAACGGCACCAGCATCAATCCGGATCTG GTGATTGACCTGACGGGTATCGGCGGTGTTAGTCCGGATCTGATTTCCAAATTCA ATCCGAAAGTTCTGATCGTCGAAGATCCGAAAGGCAACCACGACAAAGGTATCT CTAAAATCGATAACACCGACAAACGTCTGTGCGTGGGCGCGAAAAAAGGTGTTC TGAAAACCTATCGCAGCTCTAAATTTAGCAAAACGTCTGGCACCATGACCCTGGT GGTGGATATTATCATGGACTCATGTCGCGAAATTAACGAACTGGATTCGGTTCTG TATACCATCCCGAATCTGAAATACTTTCAGGGTACGGTCTTCCATGAGAAAAACG TGAAAAAATTCCTGACCGAACTGAATATGTCCGCCATTACCGTTAGTTCCATCGA TCACGTCGAATACGAACTGGAAGAAATCCTGTCAAAAAACATCAGCCGTGTGGA CTCGTTCGTGAAAGAATTTGTCGAC

\section{The S233A mutant of HcgC:}


CATATGAACTACGGCATTACCGAAAGCGTGAAAACGACCCGCAGCAAAATCAAA ATCAAAGATATTGTGTCCGATGTGGTGGAAAAGAAAGCGAACGCCATCAAATAT TTTCTGGAAGGCGAAGAATTTAAACAGGCAATTGTGTTTGGCGCTTACCTGTCAG GTTCGTATATCGCGTACTCACTGCTGAAAGATTGCGAAGAAGTCATTATCGTGGA CATTCAGCCGCATCTGAAAGATATTCTGTTCAACGACGGTATCAAATTCATGGAT CTGAACAAACTGCAACTGGAACTGCGTAACGGCACCAGCATCAATCCGGATCTG GTGATTGACCTGACGGGTATCGGCGGTGTTAGTCCGGATCTGATTTCCAAATTCA ATCCGAAAGTTCTGATCGTCGAAGATCCGAAAGGCAACCACGACAAAGGTATCT CTAAAATCGATAACACCGACAAACGTCTGTGCGTGGGCGCGAAAAAAGGTGTTC TGAAAACCTATCGCAGCTCTAAATTTAGCAAAACGTCTGGCACCATGACCCTGGT GGTGGATATTATCATGGACTCATGTCGCGAAATTAACGAACTGGATTCGGTTCTG TATACCATCCCGAATCTGAAATACTTTGAGGGTACGGTCTTCCATGAGAAAAACG TGAAAAAATTCCTGACCGAACTGAATATGTCCGCCATTACCGTTGCGTCCATCGA TCACGTCGAATACGAACTGGAAGAAATCCTGTCAAAAAACATCAGCCGTGTGGA CTCGTTCGTGAAAGAATTTGTCGAC

\section{Expression and purification}

The HcgC expression vector pET24b(+) harboring the $h c g C$ gene and its mutated genes were transferred into E.coli BL 21Star (DE3) strain (Invitrogen) and cultivated in LB medium at 37 ${ }^{\circ} \mathrm{C}$ with $50 \mu \mathrm{g} / \mathrm{mL}$ kanamycin. An over-night grown pre-culture $(50 \mathrm{ml})$ was inoculated into 2liter LB medium in 5-liter Erlenmeyer flask, which was stirred (500 rpm) using a stirrer bar. When $\mathrm{OD}_{600}$ of the culture reached to $0.8-1.0,1-\mathrm{mM}$ IPTG (final concentration) was added to the medium to induce the production of the $\mathrm{HcgC}$ protein. After $4-6$ hours cultivation at $37^{\circ} \mathrm{C}$, the $E$. coli cells were harvested by centrifugation at $15,000 \times \mathrm{g}$ for $40 \mathrm{~min}$ at $4{ }^{\circ} \mathrm{C}$. The harvested cells were stored at $-75^{\circ} \mathrm{C}$ before use. Protein purification was performed under the 
aerobic condition. The frozen cell was suspended in the $45-\mathrm{mL}$ of $50 \mathrm{mM}$ Tris/ $\mathrm{HCl} \mathrm{pH} \mathrm{7.0,20}$ $\mathrm{mM}$ imidazole, $250 \mathrm{mM} \mathrm{KCl}$ (buffer A) and disrupted on ice by sonication (Bandelin, 200W) using KE76 tip with $70 \%$ power with $50 \%$ cycle for 1 min (10 times with 1 min intervals). The crude cell extract was centrifuged at $18,000 \times \mathrm{g}$ for $40 \mathrm{~min}$ at $4{ }^{\circ} \mathrm{C}$. The supernatant was loaded onto a HisTrap HP column, which is packed with Ni Sepharose High Performance, equilibrated with buffer A. Target protein $\mathrm{HcgC}$ was eluted with linear gradient (0-100\%) of $50 \mathrm{mM}$ Tris/ $\mathrm{HCl} \mathrm{pH} 7.0$ containing $250-\mathrm{mM} \mathrm{NaCl}$ and $500-\mathrm{mM}$ imidazole (buffer B). The collected fractions were concentrated using ultrafilter (10 kDa cut-off filter, Millipore) to a final concentration of around $30 \mathrm{mg} / \mathrm{mL}$ and then stored at $-75^{\circ} \mathrm{C}$. Protein concentration was determined by the Bradford method using InstantBlue staining-solution from Expedeon. The mutated proteins were purified with the same method as the wild-type HcgC protein.

\section{Enzyme activity assay}

The HcgC enzyme-reaction solution contained 10 mM MOPS/KOH pH 7.0, 1.0 mM SAM, variable concentrations of pyridinol $2(0-0.2 \mathrm{mM})$ and $1.0 \mu \mathrm{M}$ HcgC or $1-10 \mu \mathrm{M}$ mutated enzyme. The enzyme reaction was performed aerobically under air at $37^{\circ} \mathrm{C}$. The reaction was started by addition of the enzyme solution. Product formed was determined using HPLC (JASCO) equipped with the Polar RP 80A column (Phenomenex). The reaction solution (100 $\mu 1)$ was filtrated using $0.2-\mu \mathrm{m}$ pore-size filter and $20 \mu \mathrm{l}$ was loaded to the column equilibrated with $\mathrm{H}_{2} \mathrm{O}$ (pH adjusted to 4.0 with $\mathrm{HCl}$ ). Then, the column was washed with $2.5 \mathrm{ml} \mathrm{H}_{2} \mathrm{O}$ (pH 4.0) and then eluted with $0-100 \%$ methanol gradient in a $12.5 \mathrm{ml}$ elution volume. The flow rate was $0.5 \mathrm{ml} / \mathrm{min}$. The substrate and product were eluted at around $76 \%$ and $80 \%$ methanol, respectively. As the substrate $\mathbf{2}$ and product $\mathbf{3}$ have almost the same absorbance at $288 \mathrm{~nm}$, the conversion rate from substrate to product was calculated from the ratio of [area of 3] / [total area of 2 and 3]. ${ }^{[1]}$ 


\section{Crystallization}

Crystallization of $\mathrm{HcgC}$ were performed under air. The buffer of $\mathrm{HcgC}$ was exchanged with $10 \mathrm{mM}$ MOPS/KOH pH 7.0 by concentration-dilution cycles (three times) of the protein solution using 10-kDa cut filter and finally concentrated to $\sim 5 \mathrm{mg} / \mathrm{ml}$. The $96 / 24$-well crystal plate, Combiclover Junior (Jena Bioscience), was used for crystallization. The drop consists of $1 \mu \mathrm{l}$ of enzyme solution containing $\sim 5 \mathrm{mg} / \mathrm{ml} \mathrm{HcgC,} 2 \mathrm{mM}$ pyridinol 2 and $2 \mathrm{mM}$ SAM or SAH and $1 \mu \mathrm{l}$ of the reservoir solution. The first hits were obtained in a reservoir solution containing $100 \mathrm{mM}$ Tris/HCl pH 8.5, 40\% polyethylene glycol (PEG) 400 and $200 \mathrm{mM}$ lithium sulfate $\left(\mathrm{Li}_{2} \mathrm{SO}_{4}\right)$, and $100 \mathrm{mM}$ HEPES/NaOH pH 7.5, $0.2 \mathrm{M} \mathrm{NaCl}$, and 35\% MPD (2methyl-2,4-pentanediol) within several weeks. Co-crystals of $\mathrm{HcgC}$ with SAM and pyridinol came from a crystallization solution containing 50\% v/v PEG 400, $100 \mathrm{mM} \mathrm{Na}$ acetate $\mathrm{pH} 4.5$, $200 \mathrm{mM} \mathrm{Li}_{2} \mathrm{SO}_{4}$. HcgC cocrystallized with $\mathrm{SAH}$ and pyridinol came from a crystallization solution containing 40\% v/v PEG 400, $100 \mathrm{mM}$ Tris/HCl pH 8.5 and $200 \mathrm{mM} \mathrm{Li}_{2} \mathrm{SO}_{4}$. The freshly fished crystals (growth after 2 days) were obtained from the drops containing 2-mM SAH and 2-mM pyridinol in $100 \mathrm{mM}$ HEPES/NaOH pH 7.0, 0.1 M NaCl, and 30\% MPD. Crystals of the $\mathrm{HcgC}$ apoenzyme were obtained from a solution containing $100 \mathrm{mM}$ HEPES/NaOH pH 7.5, 0.1 M NaCl, and 30\% MPD. The apoenzyme crystals were soaked overnight in the crystallization solution, which contained $2 \mathrm{mM} \mathrm{SAH}$ and $3 \mathrm{mM}$ pyridinol 2.

\section{Structure analysis}

The crystals were cryo-protected by soaking with $30 \%$ glycerol (v/v) in the crystallization solution for 3-5 seconds. The diffraction experiments were performed at $100 \mathrm{~K}$ on beamline X10SA equipped with a PILATUS 6M detector at the Swiss Light Source (Villigen). The data were processed with $\mathrm{XDS}^{[2]}$ and scaled with SCALA from the ccp4 suite. ${ }^{[3]}$ The structure was 
determined with $\mathrm{PHASER}^{[4]}$ using the HcgC model from Methanocaldococcus jannaschii in complex with SAM (PDB code 2JJF) as a template. The molecular replacement calculation succeeded by using separately the $\mathrm{N}$ and $\mathrm{C}$-terminal domain instead of the complete polypeptide chain. The model was manually constructed with $\operatorname{COOT}^{[5]}$ and refined by PHENIX. ${ }^{[4]}$ The final model was validated by using the MolProbity server (http://molprobity.biochem.duke.edu). ${ }^{[6]}$ Data collection and refinement statistics of the model are listed in Table S2. The figures were generated and rendered with PyMOL (Version 1.5, Schrödinger, LLC). 
a)

b)

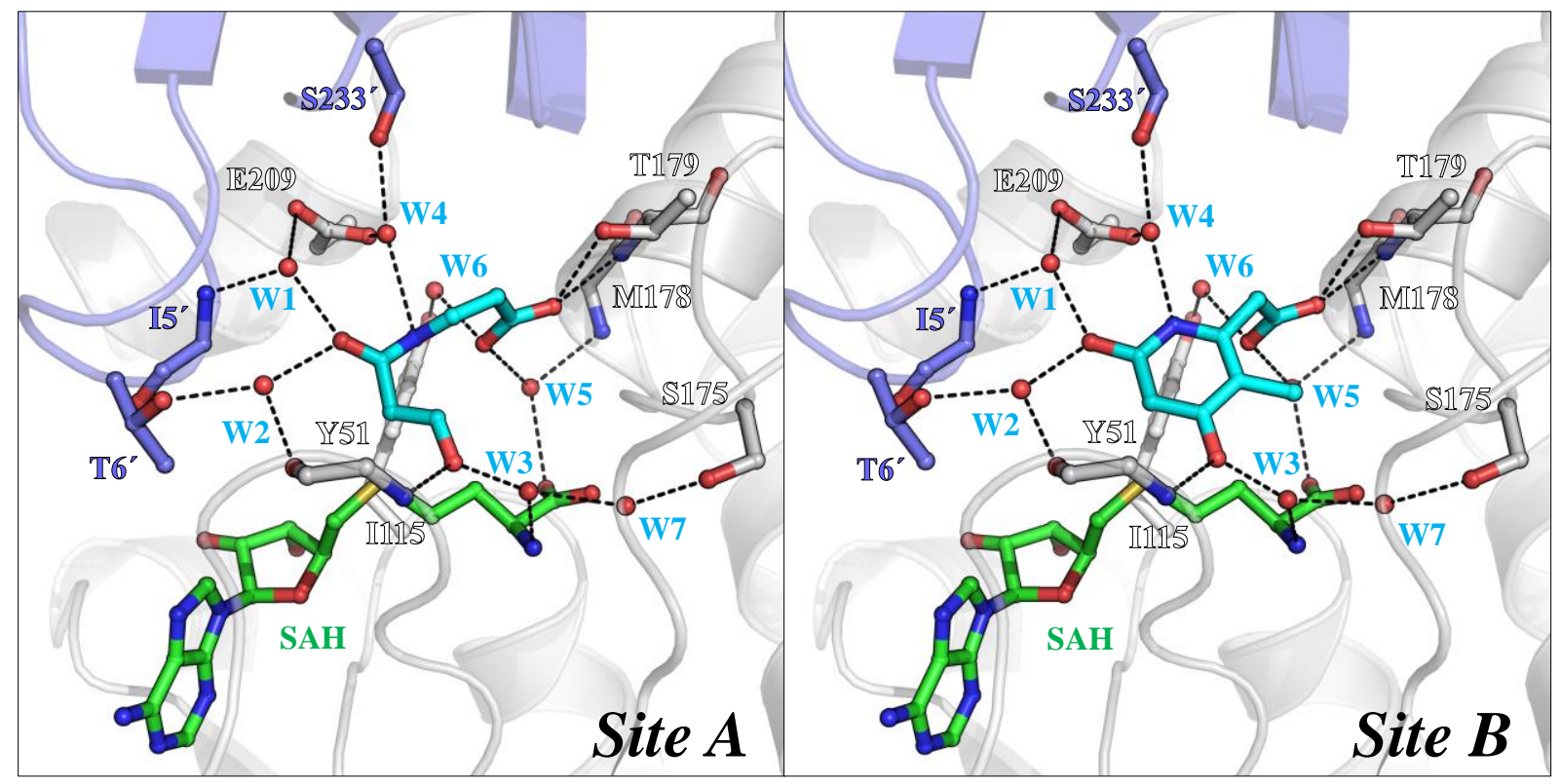

c)

d)

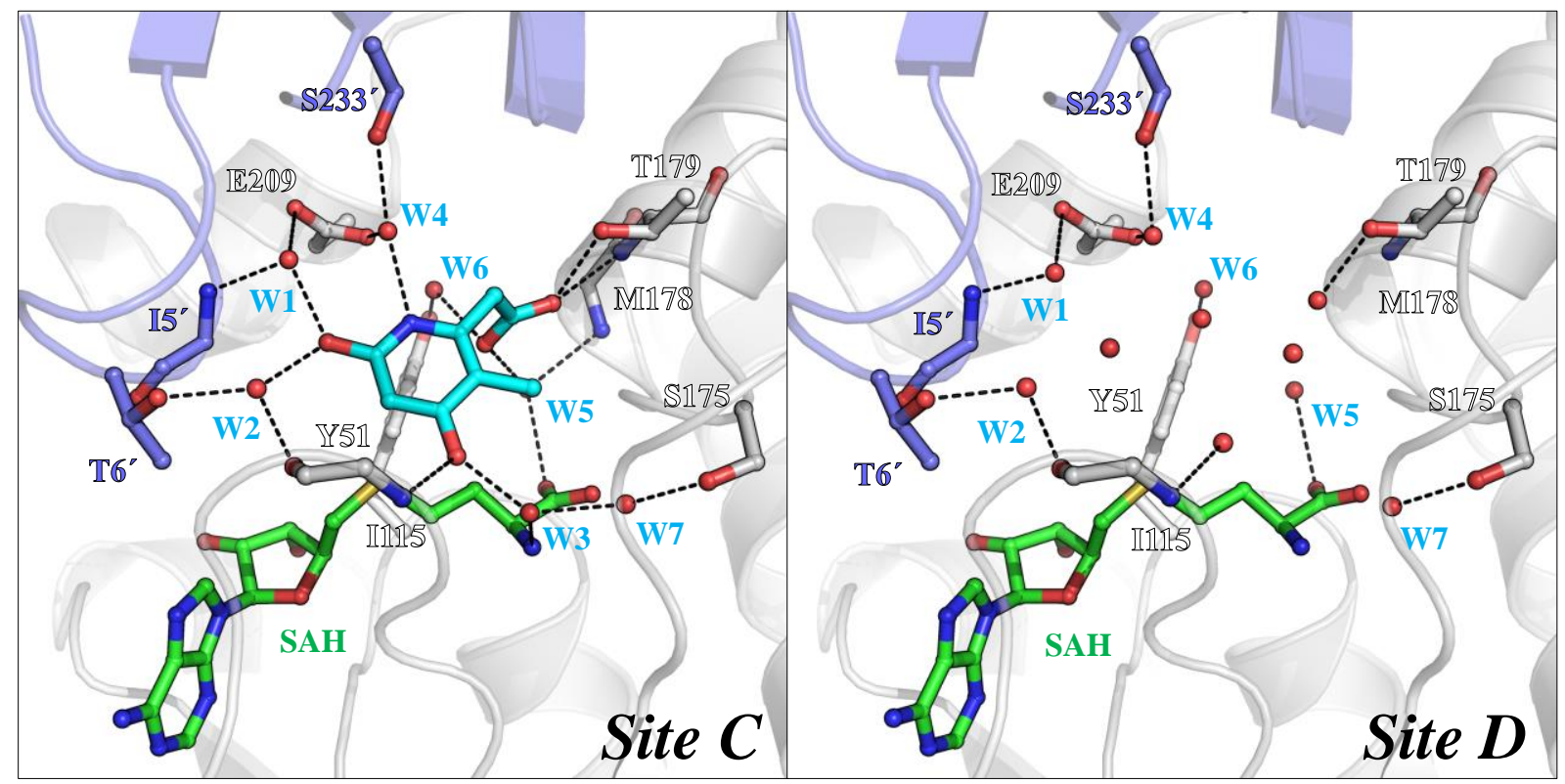

Figure S1. Active sites of the four monomers in the $\mathrm{HcgC}$ homotetramer. (a) SAH and a linear compound, (b) SAH and 2, (c) SAH and a mixture of 2 and a linear compound and (d) SAH. 

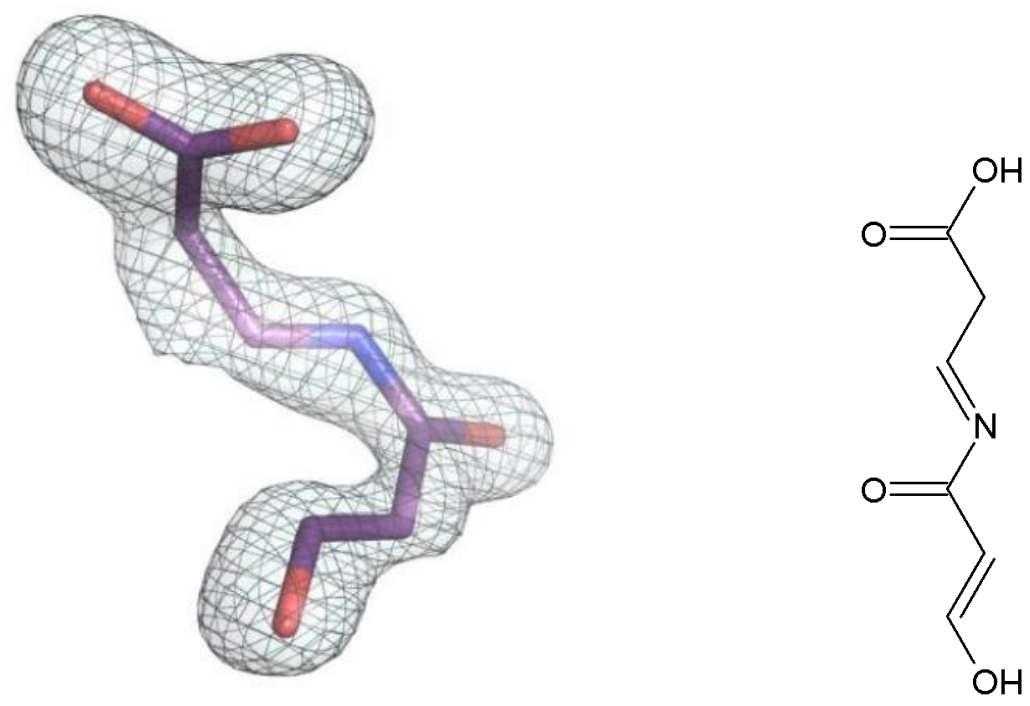

Figure S2. The linear compound found in one of the active site of the tetrameric HcgC. The chemical structure was estimated from the structure of $\mathbf{2}$ and the interactions with amino acids. The $2 F_{\mathrm{o}}-F_{\mathrm{c}}$ map is contoured at $1.5 \sigma$. 


\section{a}

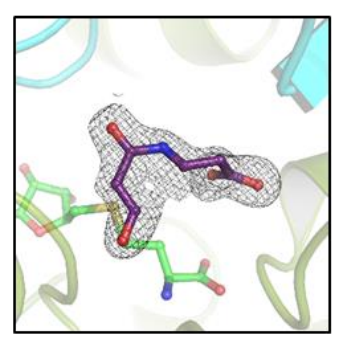

Chain A

b

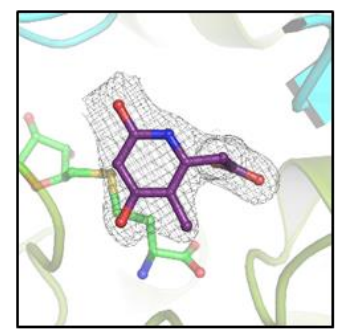

Chain A

C

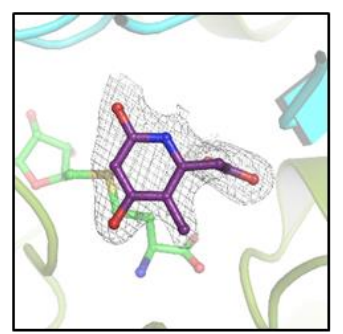

Chain A

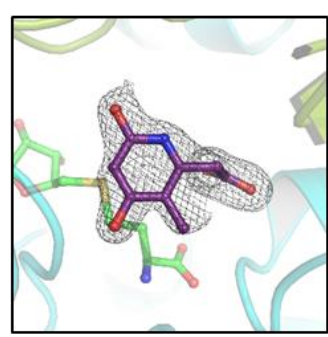

Chain B

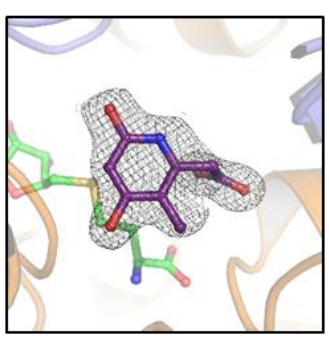

Chain C

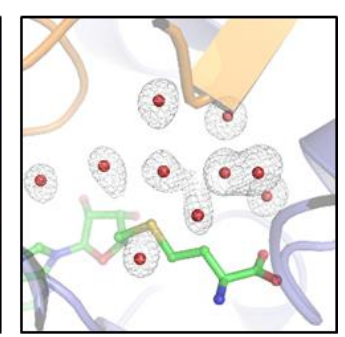

Chain D

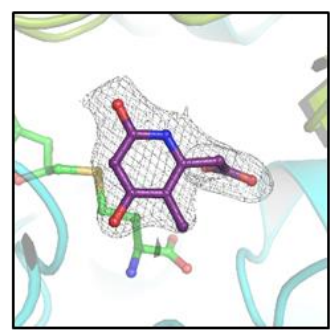

Chain B

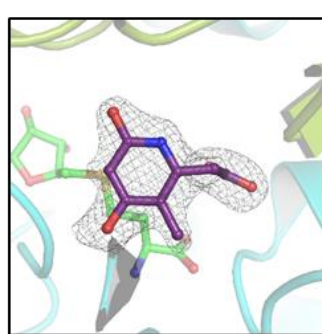

Chain B

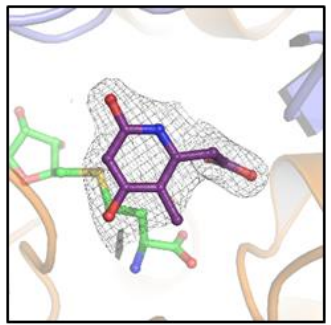

Chain C

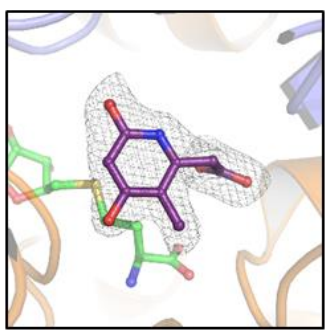

Chain C

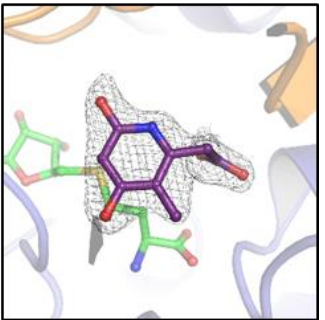

Chain D

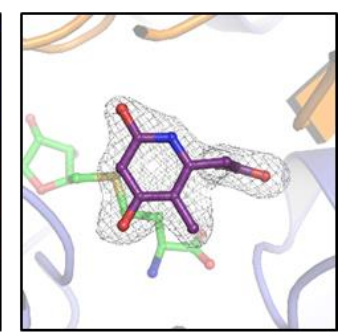

Chain D

Figure S3. Active site structure of $\mathrm{HcgC}$ from M. maripaludis in complex with SAH and pyridinol 2. The four active sites of the dimer of homodimer (chain A-D) are shown in the panels. (a) Structure from "old” crystals containing SAH and pyridinol in solution $(1.7 \AA$ resolution). (b) Structure of the crystal freshly fished after two days within less than two days (2.0 A resolution). (c) Structure of the HcgC-apoenzyme crystal soaked with the substrates (2.05 Å resolution). 
[Methanococcus_maripaludis]

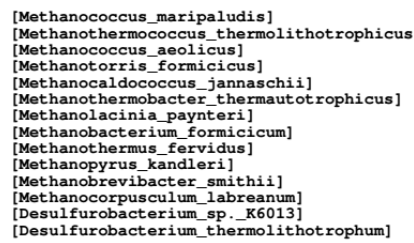

[Methanococcus_maripaludis]

[Methanococcus_maripaludis]
[Methanothermococcus_thermolithotrophicus] [Methanococcus_aeolicus]
[Methanotorris_formicicus] [Methanocaldococcus_jannaschii] Methanolacinia_paynteri] Methanothermus_fervidus] [Methanopyrus_kandleri] [Methanocorpusculum_labreanum]
[Desulfurobacterium_sp._K6013]
[Desulfurobacterium_thermolithotrophum]

[Methanococcus_maripaludis]
[Methanothermococcus_thermolithotrophicus] Methanococcus_aeolicus] Methanotorris_formicicus] Methanothermobacter_thermaut otrophicus] Methanolacinia_paynteri]
(Methanobacterium formicic [Methanothermus_fervidus] [Methanopyrus_kandleri 1 ]
[Methanobrevibacter_smithii] [Methanobrevibacter_smithii]
[Methanocorpusculum_labreanum]
[Desulfurobacterium_sp._K6013]

[Methanococcus_maripaludis]

[Methanococcus_maripaludis]
[Methanothermococcus_thermolithotrophicus] Methanococcus_aeolicus] [Methanotorris_formicicus]
[Methanocaldococcus_jannaschii] Methanothermobacter_thermau [Methanolacinia_paynteric
(Methanobacterium_formicicum] Methanothermus_fervidus Methanopyrus_kandleri 1 ]
Methanobrevibacter_smithii [Methanocorpusculum_labreanum [Methanocorpusculum__labreanum]
[Desulfurobacterium_sp._K6013]
[Desulfurobacterium_thermolithotrophum

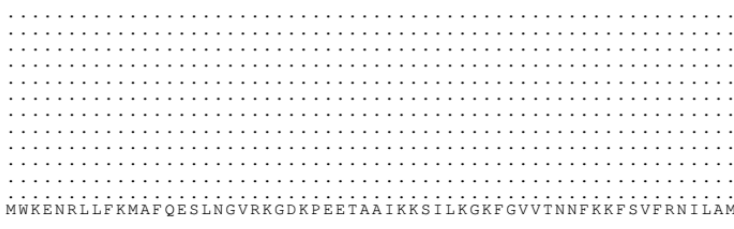

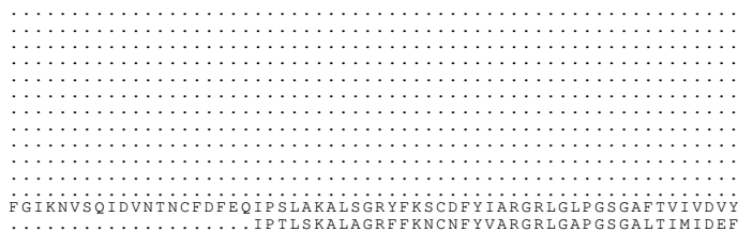
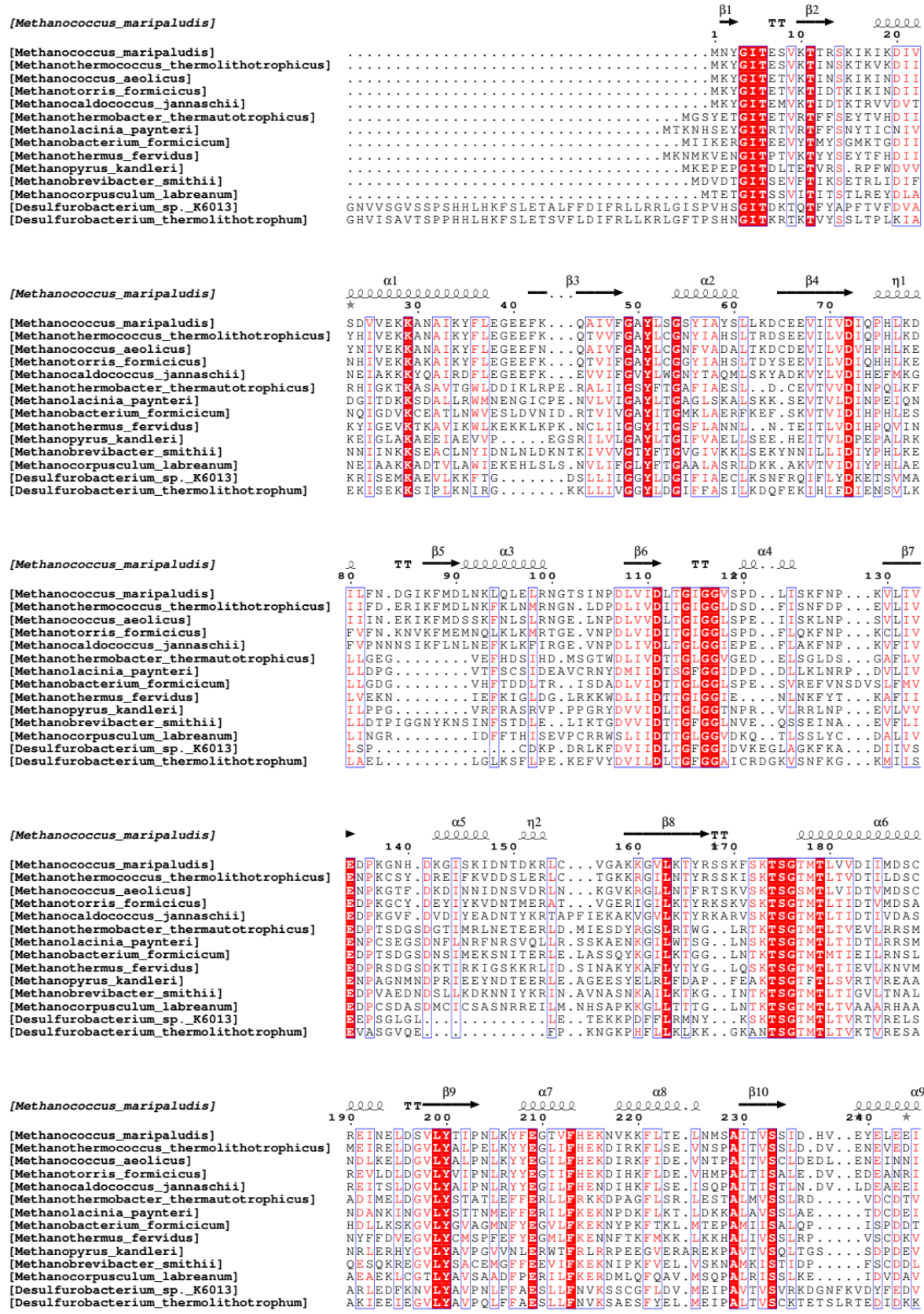
[Methanococcus_maripaludis]

[Methanococcus_maripaludis]
[Methanothermococcus_thermolithotrophicus] [Methanococcus_aeolicus]

Methanotorris_formicicus]

[Methanothermobacter_thermautotrophicus]

[Methanolacinia_paynteri]

Methanobacterium_formicicum

[Methanopyrus_kandleri]

[Methanobrevibacter_smithii]

[Dethanocorpusculam_labreanum]

[Desulfurobacterium_thermolithot rophum] $\underset{260}{\stackrel{3}{2}}$

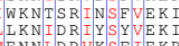

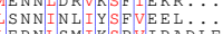

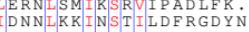

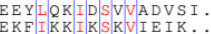

GEVIDEIIFEIRER $\ldots$

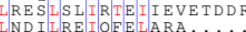

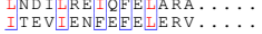

Figure S4. Comparison of primary structures of $\mathrm{HcgC}$. 


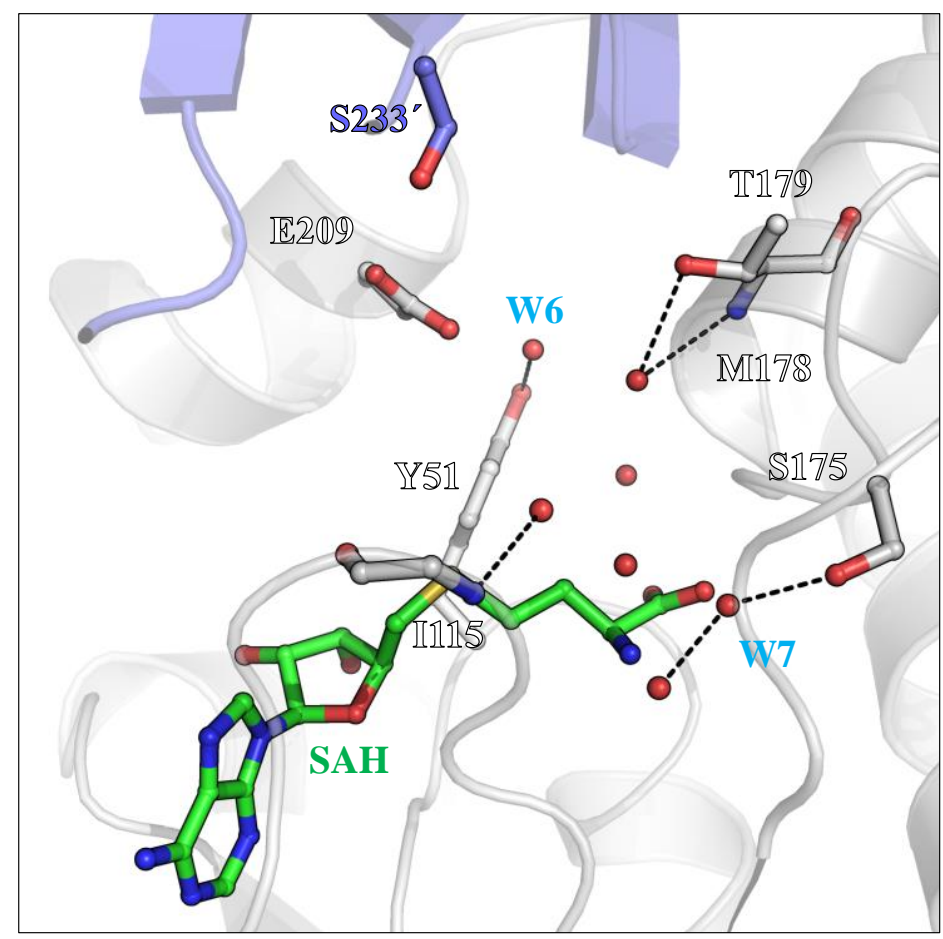

Figure S5. The active-site structure of $\mathrm{HcgC}$ crystallized in the presence of SAM and 2. In the crystallization drop, SAM and substrate $\mathbf{2}$ converted to SAH and the methylated product $\mathbf{3}$ by HcgC. Accordingly, HcgC is active under the crystallization conditions. 

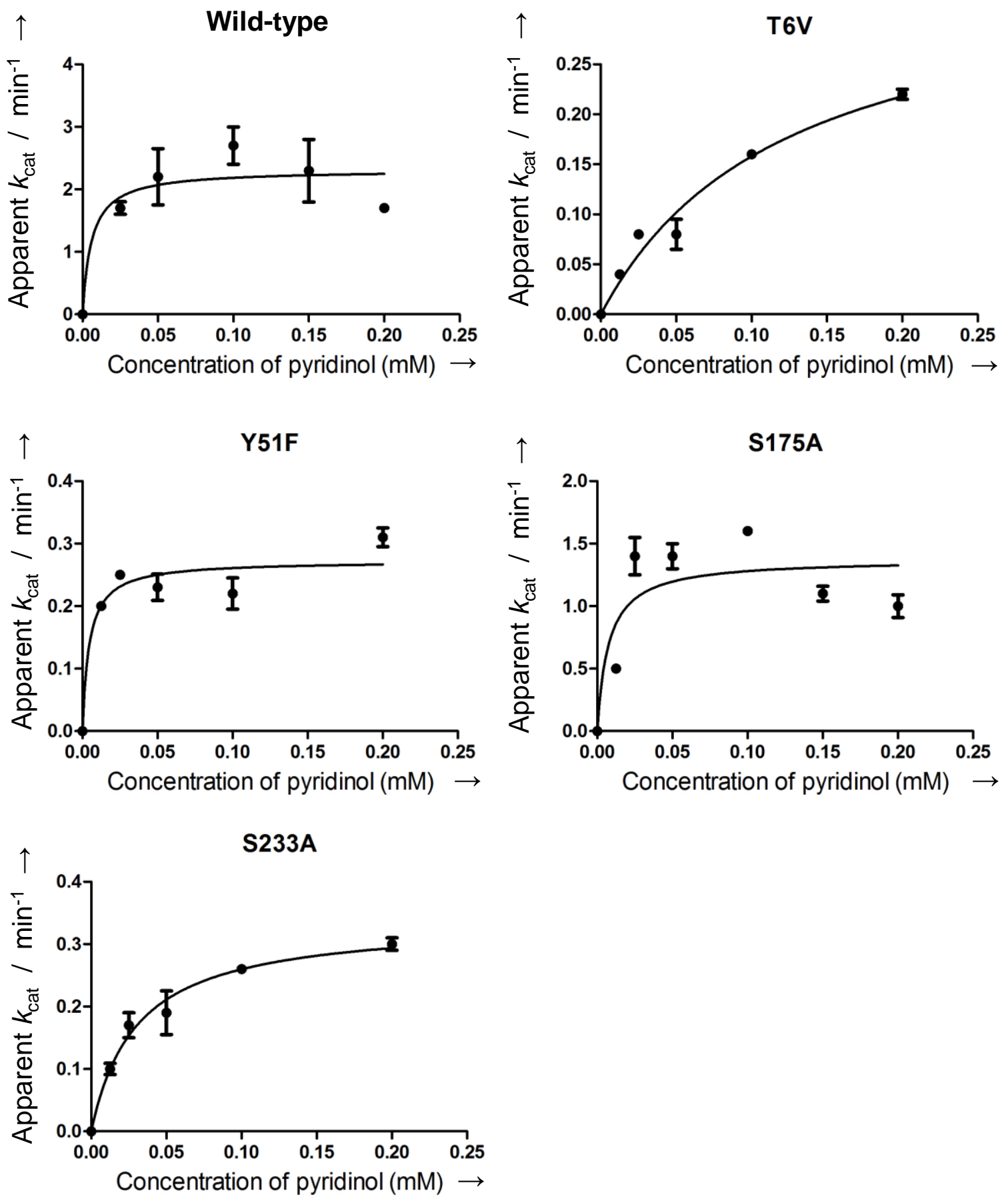

Figure S6. Kinetic data of the wild-type and mutated enzymes. The standard error of at least three measurements was calculated with the Standard Error Calculator. The assay mixture contained $1 \mathrm{mM}$ SAM and variable concentrations of substrate 2. 
Table 1. Statistics of the crystal structure analysis.

\begin{tabular}{|c|c|c|c|c|}
\hline & $\begin{array}{c}\text { HcgC } \\
\text { co-crystallized } \\
\text { with } \\
\text { SAM+pyridinol } \\
\end{array}$ & $\begin{array}{c}\text { HcgC } \\
\text { co-crystallized } \\
\text { with } \\
\text { SAH+pyridinol } \\
\end{array}$ & $\begin{array}{c}\text { HcgC freshly } \\
\text { co-crystallized } \\
\text { with } \\
\text { SAH+pyridinol } \\
\end{array}$ & $\begin{array}{c}\text { HcgC apo } \\
\text { soaked with } \\
\text { SAH+pyridinol }\end{array}$ \\
\hline \multicolumn{5}{|l|}{ Data collection } \\
\hline Wavelength $(\AA)$ & 0.99999 & 0.99999 & 1.00001 & 1.00001 \\
\hline Space group & $P 2_{1}$ & $P 2_{1}$ & $P 2_{1}$ & $P 2_{1}$ \\
\hline Resolution $(\AA)$ & $\begin{array}{c}50-1.75 \\
(1.84-1.75)\end{array}$ & $\begin{array}{c}50-1.70 \\
(1.79-1.70)\end{array}$ & $\begin{array}{c}50-2.10 \\
(2.21-2.10)\end{array}$ & $\begin{array}{c}50-2.05 \\
(2.16-2.05)\end{array}$ \\
\hline \multicolumn{5}{|l|}{ Cell dimensions } \\
\hline $\mathrm{a}, \mathrm{b}, \mathrm{c}(\AA)$ & $73.2,77.7,101.0$ & $72.7,83.0,96.1$ & $71.9,83.5,100.0$ & $72.6,81.0,97.8$ \\
\hline$\alpha, \beta, \gamma\left({ }^{\circ}\right)$ & $90.0,110.9,90.0$ & $90.0,108.0,90.0$ & $90.0,109.5,90.0$ & $90.0,108.2,90.0$ \\
\hline $\mathrm{R}_{\text {merge }}(\%)^{\mathrm{a}}$ & $8.2(64.6)$ & $7.1(56.2)$ & $5.4(54.4)$ & $10.3(71.7)$ \\
\hline $\mathrm{R}_{\text {pim }}(\%)^{\mathrm{a}}$ & $4.1(33.1)$ & $4.3(33.4)$ & $3.9(41.2)$ & $7.2(49.8)$ \\
\hline $\mathrm{CC}_{1 / 2}^{\mathrm{a}}$ & $99.7(42.1)$ & $99.7(58.9)$ & $99.8(80.4)$ & $99.3(60.0)$ \\
\hline $\mathrm{I} / \sigma_{I}^{\mathrm{a}}$ & $9.7(2.2)$ & $9.5(2.1)$ & $11.5(1.7)$ & $6.2(1.4)$ \\
\hline Completeness (\%) ${ }^{\mathrm{a}}$ & $99.7(100.0)$ & $99.6(99.9)$ & $97.9(98.8)$ & $96.7(98.0)$ \\
\hline Redundancy ${ }^{\mathrm{a}}$ & $4.8(4.7)$ & $3.7(3.8)$ & $2.6(2.5)$ & $2.9(2.9)$ \\
\hline $\begin{array}{l}\text { Number of unique } \\
\text { reflections }\end{array}$ & $106203(15490)$ & 119006 (17367) & 63739 (9372) & $65248(9601)$ \\
\hline \multicolumn{5}{|l|}{ Refinement } \\
\hline Resolution $(\AA)$ & $50.0-1.75$ & $50.0-1.70$ & $50.0-2.10$ & $30.0-2.05$ \\
\hline Number of reflections & 106177 & 118942 & 63705 & 65131 \\
\hline $\mathrm{R}_{\text {work }} / \mathrm{R}_{\text {free }}{ }^{\mathrm{b}}(\%)$ & $16.7 / 19.3$ & 15.9 / 19.2 & $20.2 / 23.3$ & $19.7 / 23.6$ \\
\hline \multicolumn{5}{|l|}{ Number of atoms } \\
\hline Protein & 8280 & 8359 & 8302 & 8263 \\
\hline Ligands/ions & 200 & 205 & 208 & 212 \\
\hline Solvent & 699 & 839 & 522 & 470 \\
\hline Mean B-value $\left(\AA^{2}\right)$ & 38.0 & 36.9 & 56.2 & 47.8 \\
\hline $\begin{array}{l}\text { Molprobity clash } \\
\text { score, all atoms }\end{array}$ & $\begin{array}{c}1.3 \\
\left(99^{\text {th }} \text { percentile }\right)\end{array}$ & $\begin{array}{c}1.9 \\
\left(99^{\text {th }} \text { percentile }\right)\end{array}$ & $\begin{array}{c}0.7 \\
\left(100^{\text {th }} \text { percentile }\right)\end{array}$ & $\begin{array}{c}0.5 \\
\left(100^{\text {th }} \text { percentile }\right)\end{array}$ \\
\hline \multicolumn{5}{|l|}{ Ramachandran plot } \\
\hline Favored regions (\%) & $1025(99.4)$ & 1029 (98.9) & $1035(99.3)$ & 1023 (98.6) \\
\hline Outlier regions $(\%)$ & 0 & 0 & 0 & 0 \\
\hline rmsd $^{\mathrm{c}}$ bond lengths $(\AA)$ & 0.010 & 0.005 & 0.010 & 0.010 \\
\hline $\operatorname{rmsd}^{\mathrm{c}}$ bond angles $\left({ }^{\circ}\right)$ & 1.210 & 0.837 & 1.190 & 1.150 \\
\hline PDB code & $5 \mathrm{O} 4 \mathrm{H}$ & $5 \mathrm{O} 4 \mathrm{~J}$ & $504 \mathrm{M}$ & $504 \mathrm{~N}$ \\
\hline
\end{tabular}

${ }^{a}$ Values relative to the highest resolution shell are within parentheses. ${ }^{b} R_{\text {free }}$ was calculated as the $R_{\text {work }}$ for $5 \%$ of the reflections that were not included in the refinement. ${ }^{c}$ rmsd, root mean square deviation. 


\section{References for Supplementary information}

[1] T. Fujishiro, L. Bai, T. Xu, X. Xie, M. Schick, J. Kahnt, M. Rother, X. Hu, U. Ermler, S. Shima, Angew. Chem. Int. Ed. 2016, 55, 9648-9651.

[2] W. Kabsch, Acta Crystallogr. D 2010, 66, 125-132.

[3] M. D. Winn, C. C. Ballard, K. D. Cowtan, E. J. Dodson, P. Emsley, P. R. Evans, R. M. Keegan, E. B. Krissinel, A. G. W. Leslie, A. McCoy, S. J. McNicholas, G. N. Murshudov, N. S. Pannu, E. A. Potterton, H. R. Powell, R. J. Read, A. Vagin, K. S. Wilson, Acta Crystallogr. D 2011, 67, 235242.

[4] P. V. Afonine, R. W. Grosse-Kunstleve, V. B. Chen, J. J. Headd, N. W. Moriarty, J. S. Richardson, D. C. Richardson, A. Urzhumtsev, P. H. Zwart, P. D. Adams, J. Appl. Crystallogr. 2010, 43, 669-676.

[5] P. Emsley, K. Cowtan, Acta Crystallogr. D 2004, 60, 2126-2132.

[6] V. B. Chen, W. B. Arendall, J. J. Headd, D. A. Keedy, R. M. Immormino, G. J. Kapral, L. W. Murray, J. S. Richardson, D. C. Richardson, Acta Crystallogr D 2010, 66, 12-21. 